PROCEEDINGS OF THE

AMERICAN MATHEMATICAL SOCIETY

Volume 128, Number 11, Pages 3425-3433

S 0002-9939(00)05425-3

Article electronically published on May 18, 2000

\title{
COMPACT WEAKLY SYMMETRIC SPACES AND SPHERICAL PAIRS
}

\author{
H. D. NGUYỄ̃
}

(Communicated by Roe Goodman)

\begin{abstract}
Let $(G, H)$ be a spherical pair and assume that $G$ is a connected compact simple Lie group and $H$ a closed subgroup of $G$. We prove in this paper that the homogeneous manifold $G / H$ is weakly symmetric with respect to $G$ and possibly an additional fixed isometry $\mu$. It follows that M. Krämer's classification list of such spherical pairs also becomes a classification list of compact weakly symmetric spaces. In fact, our proof involves a case-by-case study of the isotropy representations of all spherical pairs on Krämer's list.
\end{abstract}

\section{INTRODUCTION}

Let $M=G / H$ be a weakly symmetric space with respect to an isometry group $G$ and possibly an additional fixed isometry $\mu$. A. Selberg was able to prove in [11] that the space of $G$-invariant differential operators on $M$ is commutative. If $G$ is connected, then this is equivalent to the property of the function space $L^{1}(H \backslash G / H)$ being commutative, i.e. $(G, H)$ is a Gelfand pair (cf. [13] and 10]), or the property of every unitary irreducible representation of $G$ having at most one $H$-fixed vector, i.e. $(G, H)$ is a spherical pair and $H$ is a called a spherical subgroup of $G$ (cf. [6] and [4, Ch. IV, Theorem 3.4).

It was proven by J. Lauret in [7 that the converse to the above statement is false, namely that not all Gelfand pairs $(G, H)$ are weakly symmetric. Lauret's result involved constructing generalized Heisenberg groups $N=G / H$ endowed with a modified Heisenberg-type metric and showing that $N$ cannot be weakly symmetric with respect to the full isometry group $I(N)$. However, we prove in this paper that the converse does hold if we assume $G$ to be a connected compact simple Lie group and consider $(G, H)$ as a spherical pair. Therefore, even in light of Lauret's counterexamples, our proof will demonstrate that it is actually quite easy to establish weak symmetry for many of the compact spherical pairs.

We now give a precise statement of our main result.

Theorem 1.1. Let $(G, H)$ be a spherical pair with $G$ a connected compact simple Lie group and $H$ a closed subgroup of $G$. Then the homogeneous manifold $M=$ $G / H$ is weakly symmetric with respect to $G$ and possibly an additional fixed isometry $\mu$.

Received by the editors April 17, 1998 and, in revised form, January 4, 1999. 2000 Mathematics Subject Classification. Primary 53C35; Secondary 32M15.

Key words and phrases. Weakly symmetric spaces, spherical pairs, Gelfand pairs. 
Our strategy for proving this theorem is as follows: if $(G, H)$ is a spherical pair, then Krämer in [6] has proven that $\left(G, H_{0}\right)$ is also a spherical pair, where $H_{0}$ is the identity component of $H$. Furthermore, $\left(G, H_{0}\right)$ must appear on his classification list given in the same paper. This allows us then to go through a caseby-case study of each pair appearing on his list and prove that the corresponding homogeneous manifold $M_{0}=G / H_{0}$ is weakly symmetric with respect to $G$ and possibly an additional fixed isometry $\mu$. This is done by understanding how the isotropy representation reverses tangent vectors in each case. From this it follows that $M=G / H$ is also weakly symmetric with respect to $G$ and $\mu$ as it has an even larger isotropy subgroup than $M_{0}$.

\section{Preliminaries}

Let $M=G / H$ be a homogeneous Riemannian manifold, $G$ a transitive group of isometries and $\mu$ a fixed isometry of $M$ (not necessarily in $G$ ) satisfying $\mu G \mu^{-1}=G$ and $\mu^{2} \in G$.

Definition 2.1. $M$ is weakly symmetric with respect to $G$ and $\mu$ if given any two points $x$ and $y$ in $M$, there exists an element $g \in G$ such that $g x=\mu y$ and $g y=\mu x$.

We now present some results that will make it easier for us to identify weakly symmetric spaces in terms of their linear isotropy representations. The first is a lemma that characterizes weak symmetry as the reversal of tangent vectors, first observed by W. Ziller [17].

Lemma 2.1 (cf. 9], Lemma 2.2). Assume that $M$ is connected and $\mu$ fixes the point $z_{o}$. Define $H$ to be the isotropy subgroup of $G$ at $z_{o}$ and $T_{z_{o}}(M)$ to be the tangent space of $M$ at $z_{o}$. Then $M$ is weakly symmetric with respect to $G$ and $\mu$ if and only if given any tangent vector $v \in T_{z_{o}}(M)$, there exists an element $h \in H$ such that $d(h \circ \mu)_{z_{o}}(v)=-v$.

As weakly symmetric spaces are inherently homogeneous manifolds, Lemma 2.1 now allows us to also view weak symmetry of $M$ in terms of the adjoint representation of $H$. We wish to develop this relationship as a next step. Let $G$ be a connected Lie group and $\theta$ an involutive automorphism of $G$. If $H$ is a compact $\theta$-invariant subgroup of $G$, then there exists a reductive composition $\mathfrak{g}=\mathfrak{h}+\mathfrak{q}$ such that $\mathfrak{q}$ is $A d_{G / H}(H)$-invariant. Here, $A d(H)=A d_{G / H}(H)$ is the adjoint representation of $H$ on $\mathfrak{q} \cong \mathfrak{g} / \mathfrak{h}$.

Definition 2.2. $(G, H, \theta)$ is called a weakly symmetric triple if given any element $X \in \mathfrak{q}$, there exists an element $h \in H$ such that $(\operatorname{Ad}(h) \circ d \theta)(X)=-X$.

Lemma 2.3. If $(G, H, \theta)$ is a weakly symmetric triple, then the homogeneous manifold $M=G / H$, equipped with any $G$-invariant Riemannian metric, is weakly symmetric with respect to $G$ and the corresponding isometry $\mu$ induced from $\theta$.

Proof. Consider the natural projection map $\pi: G \rightarrow G / H$. The automorphism $\theta$ induces an involutive diffeomorphism $\mu$ of the homogeneous manifold $G / H$ :

$$
\mu(g H)=\theta(g) H, \quad g H \in G / H .
$$


Endow $M=G / H$ with any $G$-invariant Riemannian metric. Then $G$ becomes a transitive group of isometries acting by left translations on right cosets of $H$. It follows that $\mu$ is an involutive isometry satisfying $\mu G \mu^{-1}=G$ and $\mu^{2} \in G$.

Next, we identify $T_{o}(M)$, the tangent space of $M=G / H$ at the origin $o=e K$, with $\mathfrak{q}$ via $\pi$. It follows that the isotropy action of $H$ on $T_{o}(M)$ is precisely the adjoint action of $H$ on $\mathfrak{q}$. It becomes clear that $M$ is weakly symmetric with respect to $G$ and $\mu$ as a result of Lemma 2.1

Remark 2.4. It is trivial to see that if $G / K$ is a symmetric space, where $K$ is an open subgroup of $G^{\theta}$ (the fixed point set of the corresponding involution $\theta$ ), then $(G, K, \theta)$ is a weakly symmetric triple. In fact, the adjoint representation of $K$ is not needed here to reverse tangent vectors.

Lemma 2.5. Let $\rho: \tilde{G} \rightarrow G$ be a covering homomorphism between connected Lie groups, $\tilde{H} \subset G$ a connected subgroup and $H=\rho(\tilde{H})$. Then $\tilde{M}=\tilde{G} / \tilde{H}$ is weakly symmetric with respect to $\tilde{G}$ if and only if $M=G / H$ is weakly symmetric with respect to $G$.

Proof. Both $\tilde{G}$ and $G$ have isomorphic Lie algebras $\tilde{\mathfrak{g}}$ and $\mathfrak{g}$, respectively, under $d \rho$. Similarly, both $\tilde{H}$ and $H$ have isomorphic Lie algebras $\tilde{\mathfrak{h}}$ and $\mathfrak{h}$, respectively. It follows that $T_{\tilde{o}}(\tilde{M}) \cong \tilde{\mathfrak{p}} \cong \mathfrak{p} \cong T_{o}(M)$, where $\tilde{\mathfrak{p}}$ and $\mathfrak{p}$ come from the $A d_{\tilde{G} / \tilde{H}}(\tilde{H})$ and $A d_{G / H}(H)$-invariant decompositions $\mathfrak{g}=\mathfrak{h}+\mathfrak{p}$ and $\tilde{\mathfrak{g}}=\tilde{\mathfrak{h}}+\tilde{\mathfrak{p}}$, respectively. Furthermore, given any $\tilde{h} \in \tilde{H}$ with $h=\rho(\tilde{h})$, the following diagram commutes:

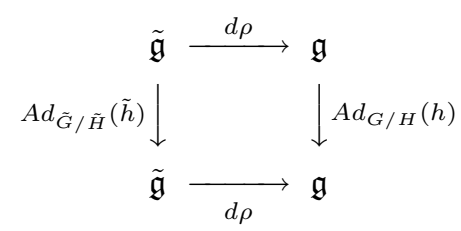

Now, fix $\tilde{v} \in \tilde{\mathfrak{p}}$ and set $v=d \rho(\tilde{v}) \in \mathfrak{p}$. Then $A d_{\tilde{G} / \tilde{H}}(\tilde{h})(\tilde{v})=-\tilde{v}$ for some $\tilde{h} \in \tilde{H}$ if and only if $A d_{G / H}(h)(v)=-v$ for some $h \in H$ with $h=\rho(\tilde{h})$.

\section{Proof of Theorem 1.1}

As described earlier in the introduction, we shall prove Theorem 1.1 by dividing the spherical pairs on Kramer's classification list into six families (categorized below) and demonstrating weak symmetry for each family:

I. $\quad$ Symmetric spaces, including $S O(8) /(S U(2) \cdot S p(2))$.

II. $\quad S^{1}$-bundles over hermitian symmetric spaces of nontube type.

III. $S U(2 n+1) / S p(n)$ and $S U(2 n+1) /(S p(n) \cdot U(1))$.

IV. Spaces of constant curvature: $G_{2} / A_{2}, S O(7) / G_{2}$ and $S O(8) / \operatorname{Spin}(7)$.

V. Spaces of Cayley-type: $S O(10) /(S O(2) \times \operatorname{Spin}(7)), S O(9) / \operatorname{Spin}(7)$ and $S O(8) / G_{2}$.

VI. $\quad S O(2 n+1) / U(n)$ and $S p(n) /(S p(n-1) \times U(1))$.

Proof of Theorem [1.1. I. If $G / K$ is a symmetric space, then it is obviously weakly symmetric with respect to the corresponding involutive isometry (the group $G$ is not needed here). We note that the homogeneous manifold $S O(8) /(S U(2) \cdot S p(2))$ appearing on Kramer's list is also a symmetric space (cf. [14], Table 3, p. 325). 
II. Let $G$ be a connected semisimple matrix Lie group with finite center and $K$ a maximal compact subgroup of $G$ such that $D=G / K$ is a hermitian symmetric space. Let $\mathfrak{g}=\mathfrak{k}+\mathfrak{p}$ be the Cartan decomposition of $\mathfrak{g}$ with respect to a Cartan involution $\sigma$, where $\mathfrak{g}$ and $\mathfrak{k}$ are the Lie algebras of $G$ and $K$, respectively. Then $\mathfrak{k}=\mathfrak{k}_{s}+\mathfrak{z} \mathfrak{k}$, where $\mathfrak{k}_{s}$ is the semisimple part of $\mathfrak{k}$ and $\mathfrak{z}_{\mathfrak{k}}$ the one-dimensional center of $\mathfrak{k}$. Let $K_{s}$ be the subgroup of $K$ with Lie algebra $\mathfrak{k}_{s}$ and $Z_{K}$ the center of $K$. Then $K_{s}$ is connected and $K=K_{s} Z_{K}^{0}$, where $Z_{K}^{0}$ is the connected component of $Z_{K}$.

Fix a maximal abelian subspace $\mathfrak{a}$ of $\mathfrak{p}$ and let $A$ and $P$ be subgroups of $G$ with Lie algebras $\mathfrak{a}$ and $\mathfrak{p}$, respectively. According to Flensted-Jensen [2], there exists an involutive automorphism $\theta$ satisfying:

Lemma 3.1. (i) $\sigma \theta=\theta \sigma$,

(ii) $\theta(a)=a^{-1}$ for all $a \in A$,

(iii) $\theta\left(K_{s}\right)=K_{s}$ and $\theta(c)=c^{-1}$ for all $c \in Z_{K}^{0}$.

At the Lie algebra level, these properties translate to

(i') $d \sigma d \theta=d \theta d \sigma$,

(ii') $d \theta(X)=-X$ for all $X \in \mathfrak{a}$,

(iii') $\theta\left(K_{s}\right)=K_{s}$ and $d \theta(X)=-X$ for all $X \in \mathfrak{z}_{K}$.

Proposition 3.2. If $G / K$ is not of tube type, then $\left(G, K_{s}, \theta\right)$ is a weakly symmetric triple.

In order to prove this proposition, we shall need some finer structure theory about hermitian symmetric spaces due to Wolf and Korányi [5]. With their permission, we also present some unpublished results of theirs obtained in the early 1980's [16].

Extend $\mathfrak{a}$ to a Cartan subalgebra $\mathfrak{h}$ of $\mathfrak{g}$ and let $Z^{J} \in \mathfrak{z} \mathfrak{k}$ be the element which gives $\mathfrak{p}$ a complex structure $J$ corresponding to the root system for $(\mathfrak{g}, \mathfrak{h})$ as described in [5]. We then split $Z^{J}=Z^{0}+Z^{\prime}$, where $Z^{0}$ defines the complex structure on the polydisc or the polysphere inside $G / K$ and $Z^{\prime}$ is an element inside $\mathfrak{k}$ which centralizes $\mathfrak{a}$.

Lemma $3.3([5]) . G / K$ is not of tube type if and only if $Z^{\prime} \neq 0$.

Lemma $3.4([16])$. Let $S^{\prime}=\left\{\exp t Z^{\prime}: t \in \mathbb{R}\right\}$. If $G / K$ is not of tube type, then

(i) $Z^{\prime} \notin \mathfrak{k}_{s}$,

(ii) $K_{s} S^{\prime}=K=S^{\prime} K_{s}$.

Proof. (i) We first note that $Z^{J} \perp \mathfrak{k}_{s}$. Then $Z^{0} \perp Z^{\prime}$ because $Z^{\prime}$ centralizes $\mathfrak{a}$ and $Z^{0} \in[\mathfrak{a}, J \mathfrak{a}] \subset \operatorname{ad}(\mathfrak{a}) \mathfrak{g}$. If $Z^{\prime} \in \mathfrak{k}_{s}$, then $0=\left\langle Z^{J}, Z^{\prime}\right\rangle=\left\langle Z^{\prime}, Z^{\prime}\right\rangle$ and forces $Z^{\prime}=0$ (here $\langle\cdot, \cdot\rangle$ is the Killing form on $\mathfrak{g}$ ).

(ii) As $Z^{\prime} \notin \mathfrak{k}_{s}$, the circle group $S^{\prime}$ acts nontrivially on the circle $K / K_{s}$. Therefore, $S^{\prime}$ is transitive there and hence $S^{\prime} K_{s}=K$. Use the map $k \mapsto k^{-1}$ to obtain $K_{s} S^{\prime}=K$.

Lemma 3.5. If $G / K$ is not of tube type, then $\operatorname{Ad}\left(K_{s}\right)(\mathfrak{a})=\mathfrak{p}$.

Proof. It is a standard result that $A d(K)(\mathfrak{a})=\mathfrak{p}$. Now write $K=K_{s} S^{\prime}$ and use the fact that $S^{\prime}$ centralizes $\mathfrak{a}$ to see that our lemma immediately follows.

Proof (second part of Proposition 3.2). Decompose $\mathfrak{g}=\mathfrak{k}_{s}+\mathfrak{z}_{\mathfrak{k}}+\mathfrak{p}$. Write any element $X \in \mathfrak{z} \mathfrak{k}+\mathfrak{p}$ as $X=Z+Y$ with $Z \in \mathfrak{z} \mathfrak{k}$ and $Y \in \mathfrak{p}$. By Lemma 3.5 we can write $Y=\operatorname{Ad}(h)(W)$ with $h \in K_{s}$ and $W \in \mathfrak{a}$. Then

$$
d \theta(Y)=A d(\theta(h))(-W)
$$


Set $k=h \theta(h)$. Now use the fact that the adjoint action of $K$ commutes with $J$ and is trivial on $\mathfrak{z}_{\mathfrak{z}}$ to check

$$
(A d(k) \circ d \theta)(X)=-X .
$$

This proves that $\left(G, K_{s}, \theta\right)$ is a weakly symmetric triple.

III. We fix $G=S U(2 n+1), K=S(U(2 n) \times U(1))$ and $K_{s}=S U(2 n)$ so that $G / K$ is complex projective space $\mathbb{C} P^{2 n}$. We choose a Cartan involution $\sigma$ so that $G$ and $K$ have the following form as matrices (see Helgason [3]):

$$
\begin{aligned}
& G=\left\{g \in S L(2 n+1, \mathbb{C}):{ }^{t} g I_{p, q} \bar{g}=I_{p, q} \text { and } \operatorname{det} g=1\right\}, \quad I_{p, q}=\left(\begin{array}{cc}
I_{2 n} & 0 \\
0 & -1
\end{array}\right), \\
& K=\left\{\left(\begin{array}{cc}
A & 0 \\
0 & D
\end{array}\right) \in G: A \in U(2 n), D \in U(1) \text { and } \operatorname{det} A \operatorname{det} D=1\right\}, \\
& K_{s}=\left\{\left(\begin{array}{cc}
A & 0 \\
0 & 1
\end{array}\right) \in G: A \in S U(2 n)\right\}, \\
& Z_{K}^{0}=\left\{\left(\begin{array}{cc}
A & 0 \\
0 & e^{-i 2 n \phi}
\end{array}\right) \in K: A=\operatorname{Diag}\left(e^{i \phi}, \ldots, e^{i \phi}\right), \quad \phi \in[0,2 \pi)\right\} .
\end{aligned}
$$

Here, the tangent space $\mathfrak{p}$ can be described by

$$
\mathfrak{p}=\left\{\left(\begin{array}{cc}
0 & W \\
\bar{W}^{t} & 0
\end{array}\right) \in \mathfrak{s u}(2 n+1): W \text { a complex } 2 n \times 1 \text { matrix }\right\}
$$

and a maximal abelian subspace $\mathfrak{a} \subset \mathfrak{p}$ chosen as

$$
\mathfrak{a}=\left\{\left(\begin{array}{cc}
0 & i w \\
-i w & 0
\end{array}\right) \in \mathfrak{p}: w \in \mathbb{R}\right\}
$$

Then the corresponding involutive automorphism $\theta$ of $G$ described in Lemma 3.1 becomes such that $\theta(g)$ is complex conjugation of all the entries of $g$ as a matrix in $S L(n, \mathbb{C})$. As $G / K$ is not of tube type, $(S U(2 n+1), S U(2 n), \theta)$ is a weakly symmetric triple by Proposition 3.2 .

Let $H \cong S p(n)$ be a maximal subgroup of $K_{s}=S U(2 n)$ embedded in $G$ as follows so that $K_{s} / H$ is a symmetric space:

$$
H=\left\{\left(\begin{array}{cc}
S p(n) & 0 \\
0 & 1
\end{array}\right)\right\} \in K_{s}
$$

where

$$
S p(n)=\left\{\left(\begin{array}{cc}
A & B \\
-\bar{B} & \bar{A}
\end{array}\right): A A^{*}+B B *=I_{n}, A B^{t}=B A^{t}\right\} .
$$

Proposition 3.6. $(S U(2 n+1), S p(n), \theta)$ is a weakly symmetric triple.

Proof. We first write $\mathfrak{s u}(2 n+1)=\mathfrak{s u}(2 n)+\mathfrak{z}_{\mathfrak{k}}+\mathfrak{p}$, where $\mathfrak{s u}(2 n)=\mathfrak{k}_{s}$. Then by writing $\mathfrak{s u}(2 n)=\mathfrak{s p}(n)+\mathfrak{q}$, we can decompose it further as $\mathfrak{s u}(2 n+1)=$ $\mathfrak{s p}(n)+\mathfrak{q}+\mathfrak{z} \mathfrak{k}+\mathfrak{p}$. It now comes down to proving that given any element $X=$ $V+Z+W \in \mathfrak{q}+\mathfrak{z} \mathfrak{k}+\mathfrak{p}$ with $V, Z$ and $W$ in $\mathfrak{q}, \mathfrak{z} k$ and $\mathfrak{p}$, respectively, there exists an element $k \in S p(n)$ such that $(A d(k) \circ d \theta)(X)=-X$. 
The Lie subalgebras $\mathfrak{s p}(n)$ and $\mathfrak{q}$ of $\mathfrak{s u}(2 n)$ are defined as follows:

$$
\begin{aligned}
\mathfrak{s p}(n) & =\left\{\left(\begin{array}{ccc}
V_{1} & V_{2} & 0 \\
-\bar{V}_{2} & \bar{V}_{1} & 0 \\
0 & 0 & 0
\end{array}\right): V_{1} \in \mathfrak{u}(n), V_{2} n \times n \text { complex matrix }\right\} ; \\
\mathfrak{q} & =\left\{\left(\begin{array}{ccc}
V_{1} & V_{2} & 0 \\
\bar{V}_{2} & -\bar{V}_{1} & 0 \\
0 & 0 & 0
\end{array}\right): V_{1} \in \mathfrak{s u}(n), V_{2} \in \mathfrak{s o}(n, \mathbb{C})\right\} .
\end{aligned}
$$

We define a maximal abelian subalgebra $\mathfrak{b}$ of $\mathfrak{q}$ as follows:

$$
\mathfrak{b}=\left\{\left(\begin{array}{ccc}
D & 0 & 0 \\
0 & -\bar{D} & 0 \\
0 & 0 & 0
\end{array}\right) \in \mathfrak{q}: D=\operatorname{Diag}\left(i d_{1}, \ldots, i d_{n}\right) ; d_{1}, \ldots, d_{n} \in \mathbb{R} ; \sum_{j=1}^{n} d_{j}=0\right\}
$$

Notice that $\left(S U(2 n), S p(n),\left.\theta\right|_{S U(2 n)}\right)$ is a weakly symmetric triple since $\left.\theta\right|_{S U(2 n)}$, the restriction of $\theta$ to $S U(2 n)$, is also an involutive automorphism with $S p(n)$ a $\theta$-invariant subgroup and satisfies all the conditions of Lemma 3.1.

As $S U(2 n) / S p(n)$ is a symmetric space, we can write $V=A d(h)(U)$, where $h \in S p(n)$ and $U \in \mathfrak{b}$. Then as the adjoint action of $S p(n)$ is trivial on $\mathfrak{z} \mathfrak{k}$,

$$
A d\left(h^{-1}\right)(X)=U+Z+W^{\prime}, \quad W^{\prime} \in \mathfrak{p} .
$$

Now, let $N=N_{S p(n)}(\mathfrak{b})$ be the normalizer of $\mathfrak{b}$ in $S p(n)$, i.e.

$$
N(\mathfrak{b})=\{l \in S p(n): \operatorname{Ad}(l)(\mathfrak{b}) \subseteq \mathfrak{b}\} .
$$

According to Lemma 3.8, there exists an element $l \in N$ such that $\operatorname{Ad}(l)\left(W^{\prime}\right)=Y$ where $Y \in \mathfrak{a}$. Hence, $\operatorname{Ad}\left(l h^{-1}\right)(X)=U^{\prime}+Z+Y$ with $U^{\prime} \in \mathfrak{b}$. In other words,

$$
X=A d\left(h l^{-1}\right)\left(U^{\prime}+Z+Y\right) .
$$

As $d \theta=-\mathrm{Id}$ on $\mathfrak{b}+\mathfrak{z} \mathfrak{k}+\mathfrak{a}$, it follows that

$$
d \theta(X)=A d\left(\theta\left(h l^{-1}\right)\right)\left(-U^{\prime}-Z-Y\right) .
$$

Setting $k=h l^{-1} \theta\left(h l^{-1}\right) \in S p(n)$, we get

$$
(A d(k) \circ d \theta)(X)=-X .
$$

We also obtain as an obvious result the following corollary:

Corollary 3.7. $(S U(2 n+1), S p(n) \cdot U(1), \theta)$ is also a weakly symmetric triple.

Lemma 3.8. $\operatorname{Ad}_{G}(N)(\mathfrak{a})=\mathfrak{p}$.

Proof. It is clear that $A d_{G}(N)(\mathfrak{a}) \subset \mathfrak{p}$. We introduce some notation. Let $E_{i, j}$ denote the $n \times n$ matrix with 1 in the $i j$ entry and 0 's everywhere else, $F_{i, j}$ the $n \times n$ matrix obtained from the identity matrix by switching the $i$-th row with the $j$-th row, and $W_{j}$ the $(2 n+1) \times 1$ matrix with 1 in the $j$-th row and 0 's everywhere else. Now, decompose $\mathfrak{p}=\sum_{j=1}^{2 n} \mathfrak{a}_{j}$, where $\mathfrak{a}_{j}$ is the complex subspace spanned by the matrix

$$
\left(\begin{array}{cc}
0 & W_{j} \\
W_{j}^{t} & 0
\end{array}\right)
$$


The following assertions can be easily verified:

(i) Since $N$ contains the diagonal matrices in $S p(n)$, it follows that $\operatorname{Ad}(N)(\mathfrak{a}) \supset$ $\mathfrak{a}_{1}$.

(ii) Since $N$ contains matrices of the form

$$
L_{j}=\left(\begin{array}{ccc}
F_{j, j+1} & 0 & 0 \\
0 & F_{j, j+1} & 0 \\
0 & 0 & 1
\end{array}\right), \quad j=1, \ldots, n,
$$

it follows that $\operatorname{Ad}\left(L_{j}\right)\left(\mathfrak{a}_{j}\right)=\mathfrak{a}_{j+1}$ and $\operatorname{Ad}\left(L_{j}\right)\left(\mathfrak{a}_{n+j}\right)=\mathfrak{a}_{n+j+1}$.

(iii) Since $N$ contains matrices of the form

$$
\tilde{L}_{j}=\left(\begin{array}{ccc}
I_{n}-E_{j, j} & E_{j, j} & 0 \\
-E_{j, j} & I_{n}-E_{j, j} & 0 \\
0 & 0 & 1
\end{array}\right), \quad j=1, \ldots, n,
$$

it follows that $\operatorname{Ad}\left(\tilde{L}_{j}\right)\left(\mathfrak{a}_{j}\right)=\mathfrak{a}_{n+j}$.

From these assertions, we conclude that $\operatorname{Ad}(N)(\mathfrak{a})=\mathfrak{p}$. This completes the proof of the lemma and Proposition 3.6.

IV. Spaces of constant curvature:

1. $S O(8) / \operatorname{Spin}(7)$ : We have the double cover $\operatorname{Spin}(8) / \operatorname{Spin}(7) \rightarrow S O(8) / \operatorname{Spin}(7)$. Since $\operatorname{Spin}(8) / \operatorname{Spin}(7) \cong S^{7}$ is a space of constant curvature and the isotropy action is transitive on the unit tangent sphere, it follows that $\operatorname{Spin}(8) / \operatorname{Spin}(7)$ is weakly symmetric with respect $\operatorname{Spin}(8)$. Hence, $S O(8) / \operatorname{Spin}(7)$ is weakly symmetric with respect to $S O(8)$ by Lemma 2.5

2. $S O(7) / G_{2}$ : Again, we have the double cover $\operatorname{Spin}(7) / G_{2} \rightarrow S O(7) / G_{2}$. Now, $\operatorname{Spin}(7) / G_{2} \cong S^{7}$ is a space of constant curvature and therefore weakly symmetric with respect to $G_{2}$. Hence, $S O(7) / G_{2}$ is weakly symmetric with respect to $S O(7)$.

3. $G_{2} / A_{2}$ : From [15], Proposition 8.12.7, it is known that $G_{2} / A_{2}=S^{6}$ is a space of constant curvature and its isotropy action is transitive on the unit tangent sphere. Hence, $G_{2} / A_{2}$ is weakly symmetric with respect to $G_{2}$.

V. Spaces of Cayley-type:

1. $S O(10) /\left(S O(2) \times \operatorname{Spin}_{ \pm}(7)\right)$ : Following 12, Theorem 5.4, we have the decomposition

$$
\mathfrak{s o}(10) /(\mathfrak{s p i n}(7) \oplus \mathfrak{s o}(2))=\mathbb{R}^{7} \oplus\left(\mathbb{R}^{8} \otimes \mathbb{R}^{2}\right) .
$$

Here, the adjoint representation of $\operatorname{Spin}_{ \pm}(7) \times S O(2)$ on the factor $\mathbb{R}^{7}$ acts through the standard representation of $\operatorname{Spin}(7)$ on $\mathbb{R}^{7}$ (the action of $S O(2)$ is trivial) and on the factor $\mathbb{R}^{8} \otimes \mathbb{R}^{2}$ through the spin representation of $\operatorname{Spin}(7)$ on $\mathbb{R}^{8}$ and by rotation of $S O(2)$ on $\mathbb{R}^{2}$. Furthermore, if $v \in \mathbb{R}^{7}$, then the isotropy subgroup $(\operatorname{Spin}(7) \times S O(2))_{v}=S U(4) \times S O(2)$, where $S U(4)$ acts on $\mathbb{R}^{8} \cong \mathbb{C}^{4}$ by the standard action.

Therefore, given any vector $v+w \in \mathbb{R}^{7} \oplus\left(\mathbb{R}^{8} \otimes \mathbb{R}^{2}\right)$, there exists an element $k \in \operatorname{Spin}(7)$ which sends $v$ to its negative. Next, we write $w=\sum_{i=1}^{p} e_{i} \otimes f_{j}$, where $e_{i} \in \mathbb{R}^{8}$ and $f_{j} \in \mathbb{R}^{2}$. As $S O(2)$ acts by rotations, we use the rotation $e^{i \pi}$ to reverse each $f_{j}$ and therefore send $w$ to $-w$. Together, the element $\left(k, e^{i \pi}\right)$ in $\operatorname{Spin}(7) \times S O(2)$ will then send $v+w$ to $-(v+w)$. This proves weak symmetry for $S O(10) /(S O(2) \times \operatorname{Spin}(7))$.

2. $S O(9) / S p i n(7)$ and $S O(8) / G_{2}$ : According to [8], there exists double covers

$$
\operatorname{Spin}(9) / \operatorname{Spin}(7) \rightarrow \operatorname{SO}(9) / \operatorname{Spin}(7) \text { and } \operatorname{Spin}(8) / G_{2} \rightarrow \operatorname{SO}(8) / G_{2} .
$$


TABLE 1. Compact weakly symmetric spaces

\begin{tabular}{|c|c|c|c|}
\hline & \multicolumn{2}{|c|}{$M=G / H$ weakly symmetric } & \\
\hline Family & $G$ & $H$ & w.r.t. \\
\hline $\mathrm{I}$ & Symmetric s & es with involutive isometry $\mu$ & $\mu$ \\
\hline II & $\begin{array}{l}S^{1} \text {-bundles c } \\
S U(n+m) \\
S O(2 n) \\
E_{6}\end{array}$ & $\begin{array}{ll}\text { hermitian symmetric spaces: } \\
S U(m) \times S U(m) & (n>m \geq 1) \\
S U(n) & (n \geq 3, n \text { odd }) \\
D_{5} & \end{array}$ & $\begin{array}{l}G \text { and } \mu \\
G \text { and } \mu \\
G \text { and } \mu\end{array}$ \\
\hline III & $\begin{array}{l}S U(2 n+1) \\
S U(2 n+1)\end{array}$ & $\begin{array}{ll}S p(n) & (n \geq 1) \\
S p(n) \cdot U(1) & (n \geq 1)\end{array}$ & $\begin{array}{l}G \text { and } \mu \\
G \text { and } \mu\end{array}$ \\
\hline IV & $\begin{array}{l}\text { Spaces of co } \\
S O(8) \\
S O(7) \\
G_{2}\end{array}$ & $\begin{array}{l}\text { ant curvature: } \\
\operatorname{Spin}(7) \\
G_{2} \\
A_{2}\end{array}$ & $\begin{array}{l}G \\
G \\
G\end{array}$ \\
\hline $\mathrm{V}$ & $\begin{array}{l}\text { Spaces of } \mathrm{Ca} \\
S O(10) \\
S O(9) \\
S O(8)\end{array}$ & $\begin{array}{l}\text { y-type: } \\
\operatorname{SO}(2) \times \operatorname{Spin}(7) \\
\operatorname{Spin}(7) \\
G_{2}\end{array}$ & $\begin{array}{l}G \\
G \\
G\end{array}$ \\
\hline VI & $\begin{array}{l}S O(2 n+1) \\
S p(n)\end{array}$ & $\begin{array}{ll}U(n) & (n \geq 2) \\
S p(n-1) \times U(1) & (n \geq 1)\end{array}$ & $\begin{array}{l}G \\
G\end{array}$ \\
\hline
\end{tabular}

But $\operatorname{Spin}(9) / \operatorname{Spin}(7)$ and $\operatorname{Spin}(8) / G_{2}$ are already known to be weakly symmetric with respect to $\operatorname{Spin}(9)$ and $\operatorname{Spin}(8)$, respectively, from [17, pp. 357 and 361.

VI. $S O(2 n+1) / S U(n)$ and $S p(n) /(U(1) \times S p(n-1))$ : Again, these spaces have already been proven to be weakly symmetric in [17], pp. 359-360.

This concludes the proof of Theorem 1.1. Table 1 summarizes our results with appropriate descriptions of weak symmetry for each family.

\section{ACKNOWLEDGEMENTS}

The author wishes to thank W. Ziller for his generous help and guidance. Many of the arguments for the spherical pairs of constant curvature and of Cayley-type actually resulted from joint work between us. Furthermore, he has recently informed the author that D.N. Akhiezer and E.B. Vinberg have announced a similar result in [1] but within the context of spherical varieties. In fact, they prove that Theorem 1.1 holds more generally for real reductive algebraic groups $G$.

\section{REFERENCES}

[1] D.N. Akhiezer and E.B. Vinberg, Weakly symmetric spaces and spherical varieties, preprint (1998).

[2] M. Flensted-Jensen, Spherical functions on a simply connected semisimple Lie group, Amer. J. Math. 99 (1977), 341-361. MR 56:16266

[3] S. Helgason, Differential Geometry, Lie Groups, and Symmetric Spaces, Pure and Applied Mathematics 80, Academic Press, 1978. 
[4] S. Helgason, Groups and Geometric Analysis, Pure and Applied Mathematics 113, Academic Press, 1984. MR 86c:22017]

[5] A. Korányi and J.A. Wolf, Realization of hermitian symmetric spaces as generalized halfplanes, Ann. of Math. 81 (1965), 265-288. |MR 30:4980

[6] M. Krämer, Sphärische Untergruppen in kompakten zusammenhängenden Liegruppen, Compositio Math. 38 (1979) No. 2, 129-153. MR 80f:22011

[7] J. Lauret, Commutative spaces which are not weakly symmetric, Bull. London Math. Soc. 30 (1998) No. 1, 29-36. MR 99a:22016

[8] S. Murakami, Exceptional simple Lie groups and related topics in recent differential geometry, Proceedings: Differential Geometry and Topology (Tianjin 1986-87), Lecture Notes in Mathematics 1369, Springer-Verlag, 1989. MR 90g:22009

[9] H.D. Nguyêñ, Weakly symmetric spaces and bounded symmetric domains, Transform. Groups 2 (1997) No. 4, 351-374. MR 98k:53067

[10] H.D. Nguyêñ, Characterizing weakly symmetric spaces as Gelfand pairs, J. Lie Theory (to appear). CMP 99:09

[11] A. Selberg, Harmonic analysis and discontinuous groups in weakly symmetric Riemannian spaces with applications to Dirichlet series, J. Indian Math. Soc. 20 (1956), 47-87. MR 19:531g

[12] H. Tamaru, Riemannian geodesic orbit metrics on fiber bundles, preprint (1997).

[13] E.G.F. Thomas, An infinitesimal characterization of Gelfand pairs, Contemp. Math. 26 (1984), 379-385. MR 85i:22015

[14] M. Wang and W. Ziller, Symmetric spaces and strongly isotropy irreducible spaces, Math. Ann. 296 (1993), 285-326. MR 94g:53042

[15] J.A. Wolf, Spaces of Constant Curvature, McGraw-Hill, 1967.

[16] J.A. Wolf and A. Korányi, Working notes, August 1983.

[17] W. Ziller, Weakly symmetric spaces, Topics in Geometry: In Memory of Joseph D' Atri, Progress in Nonlinear Diff. Eqs. 20, Birkhauser, 1996, 355-368. MR 97c:53081

Department of Mathematics, Rowan University, Glassboro, New Jersey 08028

E-mail address: nguyen@rowan.edu 